\title{
The Effects of the Plasmon-LO Phonon Interaction on the Critical Densities of RPA Approach in a Quasi-One-Dimensional System
}

\author{
P. C. M. Machado*, F. A. P. Osório ${ }^{\dagger, \overrightarrow{+}}$, and A. N. Borges ${ }^{\dagger, *}$ \\ ${ }^{*}$ Escola de Engenharia Elétrica e de Computação, Universidade Federal de Goiás, CP 131, 74001-970 Goiânia-GO, Brazil \\ ${ }^{\dagger}$ Instituto de Física, Universidade Federal de Goiás, CP 131,74001-970 Goiânia-GO, Brazil \\ ॠNúcleo de Pesquisa em Física, Universidade Católica de Goiás, 74605-220 Goiânia-GO, Brazil
}

Received on 4 April, 2005

\begin{abstract}
In this work we have studied the electron LO phonon interaction on the pair-correlation function $g(x)$ and its dependence on the electronic density for a GaAs-AlGaAs rectangular quantum wire within the random-phase approximation (RPA). We assumed two different values of the wire width. As negative non-physical results are found for lower electronic densities and small interparticle separations in the RPA approach, we have delimited the regions where the RPA approach cannot be used for the calculation of the Q1D electron gas collective excitation.
\end{abstract}

Keywords: Plasmon-LO phonon interaction; RPA Approach; GaAs-AlGaAs rectangular quantum wire

\section{INTRODUCTION}

The interest in the investigation of electron gas coupled to longitudinal-optical (LO) phonon in low dimensional semiconductor structures has been growing motivated by the improvements in the growth techniques and by the potential technological applications of these heterostructures [1-6]. As $\mathrm{GaAs}$ and $\mathrm{AlGaAs}$ are the main materials used to fabricate these heterostructures and they are weakly polar, the polarization field of the LO phonons in such materials follows the electron motion, producing a system called polaron, resulting in a polaron gas. The strength of the polaronic interaction may be strong when the frequencies of both plasmon and phonon are comparable

The many-body effects of a quasi-one-dimensional (Q1D) electron gas present in a quantum well wire (QWW) structure have motivated a significant number of works [7-12]. The most common method used in theoretical works has been the random-phase approximation (RPA). For a GaAs quantum well wire of rectangular cross section Wendler, Haupt and Pechstedt [5] using a three subband model within the RPA, studied the coupling properties of the Q1D polaron gas and a strong interaction between different types of plasmon and the LO phonon was found. Borges, Osório, Machado and Hipólito [6] studied the behavior of the inter and intra- subband energy dispersion relation of the polaron gas, obtained within the self consistent Singwi,Tosi,Land and Sjolander theory (STLS) [13]. The STLS results are compared with the RPA results and found that the differences between the two calculation methods (STLS and RPA) are more expressive for the intrasubband plasmon (0-0) and that the short range correlation effects are more expressive when the layer thickness of the wire decreases.

As is well known, the RPA approach leads to negative nonphysical result values for the pair-correlation function $g(x)$ for lower electronic densities and small interparticle separation However, these previous works did not study the validity of the theoretical calculation methods (RPA and STLS) as a function of the polaron gas density.

We define the critical density as the density from which the RPA approach gives non-negative values for the paircorrelation function for small interparticle separation [14]. For density values lower than the critical density value, the local-field correction (LFC) must always be used.

In this work we study the effects of the electron - LO phonon interaction on the pair-correlation-function as a function of the interparticle separation for several values of the polaron gas densities. Also we study the critical densities as a function of the electron density for two QWW width with infinite potential barrier heights.

\section{THEORY}

Let us consider a rectangular Q1D quantum well wire of GaAs with width $L$ along the $x$-direction, zero thickness layer along the $z$-direction at $z=0$ and infinite potential barrier height in the $y$-direction. Following the treatment of Borges et al. [6], the screening interaction potential $V_{i j l m}\left(q_{x}, \omega\right)$, describing the electron interaction with other electrons and with the bulk LO phonon, within the RPA approach can be written as

$$
\begin{gathered}
V_{i j l m}\left(q_{x}, \omega\right)=\frac{2 e^{2}}{\varepsilon_{\infty}} \int_{0}^{\infty} d k \frac{F_{i j l m}\left(k, q_{x}\right) A(\omega)}{\sqrt{k^{2}+q_{x}^{2}}} \\
A(\omega)=\left[1+\frac{2 \omega_{L O}}{\hbar\left(\omega^{2}-\omega_{L O}^{2}\right)} \frac{\varepsilon_{\infty} \alpha\left(\hbar \omega_{L O}\right)^{2} r_{p}}{e^{2}}\right]
\end{gathered}
$$

where $F_{i j l m}\left(k, q_{x}\right)$ is the form factor, which takes into account the geometric form of the quantum wire, $\varepsilon_{\infty}$ is the highfrequency dielectric constant, $\omega_{L O}$ is the frequency of the LO phonon system, $\alpha$ is the Frölich electron-phonon coupling constant and $r_{p}$ is the polaron radius.

In the mean-field approximation, the RPA generalized response function of the system can be written as [12] 


$$
\chi_{i j l m}\left(q_{x}, \omega\right)=\frac{\chi_{l m}\left(q_{x}, \omega\right)}{\delta_{i l} \delta_{j m}-V_{i j l m}\left(q_{x}\right) \chi_{l m}\left(q_{x}, \omega\right)},
$$

where the symbol $\delta_{i j}$ is the Kronecker delta, $\chi_{l m}=P_{l m}$ if $l=m$ and $\chi_{l m}=P_{l m}+P_{m l}$ when $l \neq m . P_{l m}\left(q_{x}, \omega\right)$ is the RPA polarization function of the Q1D electron gas. From this result, the static structure factor $S$ can be obtained as [12]

$$
S_{i j l m}\left(q_{x}\right)=-\frac{\hbar}{\pi \rho} \int_{0}^{\infty} d \omega \operatorname{Im}\left[\chi_{i j l m}\left(q_{x}, \omega\right)\right],
$$

where $\rho=1 / L$ is the one-dimensional density of electrons in the system and $\operatorname{Im}[f]$ denotes the imaginary part of the function $f$. The pair-correlation function is calculated by the relation [12]

$$
g_{i j l m}(x)=1+\frac{1}{\pi \rho} \int_{0}^{\infty} d q_{x}\left(S_{i j l m}\left(q_{x}\right)-1\right) \cos \left(q_{x} x\right) .
$$

\section{RESULTS AND DISCUSSION}

The pair-correlation function for GaAs-AlGaAs quantum well wires of rectangular cross section was calculated within the random-phase approximation. Two wire widths were considered: $300 \AA$ and $500 \AA$. The potential barrier height was assumed as infinite. The effective Rydberg energy and the effective Bohr radius used in the present calculation were $R_{y}^{*}=$ $7.67 \mathrm{meV}$ and $a_{0}^{*}=86 \AA$, respectively. The other parameters were assumed as $\varepsilon_{\infty}=10.9, \eta \omega_{L O}=36.18 \mathrm{meV}$ and $\eta \omega_{T O}=$ $33.29 \mathrm{meV}$.

Figure 1 shows the intrasubband pair-correlation function $g(x)$, as a function of the dimensionless interparticle separation coordinate $x / a_{0}^{*}$ with phonon effects (solid lines) and without phonon effects (dashed lines) for three electronic density values: $\rho=7.7 \times 10^{5} \mathrm{~cm}^{-1}$ (higher lines), $\rho=6.1 \times 10^{5}$ $\mathrm{cm}^{-1}$ (intermediate lines) and $\rho=3.9 \times 10^{5} \mathrm{~cm}^{-1}$ (lower lines). As it can be seen, the presence of the phonon contributes significantly to the pair correlation function mainly in the region of small interparticle separation. The contribution of the polaronic effect in this region became $\mathrm{g}(\mathrm{x})$ more negative, showing that the RPA approach is more inadequate and the Local Field Correction must be included in the calculation to give the correct results.

Figure 2 shows the intrasubband pair-correlation function near the origin $g(x \approx 0)$, as a function of the electronic density for two wire widths, $\mathrm{L}=300 \AA$ and $\mathrm{L}=500 \AA$. The solid line at $g(x)=0$ delimits the density region where the RPA approach cannot be used. In this figure it can be seen that the critical density increases with decreasing wire width: the critical density for a QWW with wire width $\mathrm{L}=500 \AA$ is $\rho=4.5 \times 10^{5}$ $\mathrm{cm}^{-1}$, while the critical density for a QWW with wire width $\mathrm{L}=300$ ? is $\rho=6.1 \times 10^{5} \mathrm{~cm}^{-1}$.

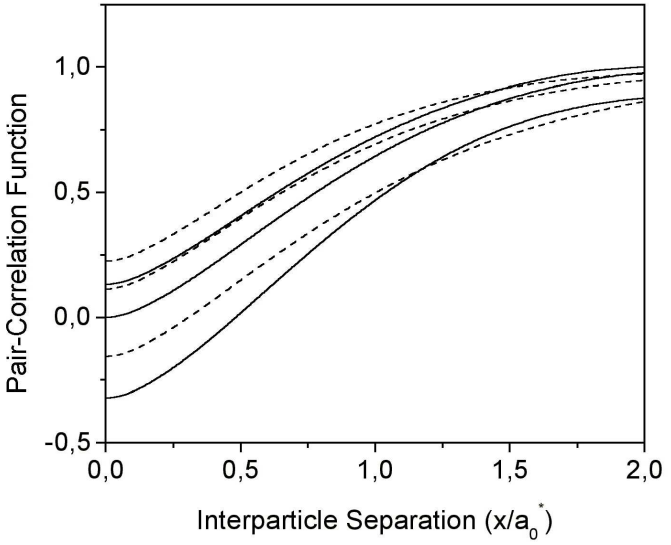

FIG. 1: The pair-correlation function calculated with (solid lines) and without phonon effects (dashed lines) for three electronic density values: $\rho=7.7 \times 10^{5} \mathrm{~cm}^{-1}$ (higher lines), $\rho=6.1 \times 10^{5} \mathrm{~cm}^{-1}$ (intermediate lines) and $\rho=3.9 \times 10^{5} \mathrm{~cm}^{-1}$ (lower lines).

Naturally the inclusion of the local field corrections, as the STLS theory, gives better theoretical results in all way, mainly as more realistic was the calculation model. The inclusion of the polaronic effects in the calculations became the system model more realistic and increases the needs of the LFC inclusion and gives an important contribution to the excitation energy that cannot be neglected [6].

However with LFC inclusion the complexity of the calculations increases very much. In this sense the results shown in Fig. 2, show the values of the electronic densities where the RPA approach gives good results. The advantage of this information is that this calculation method is very simple.

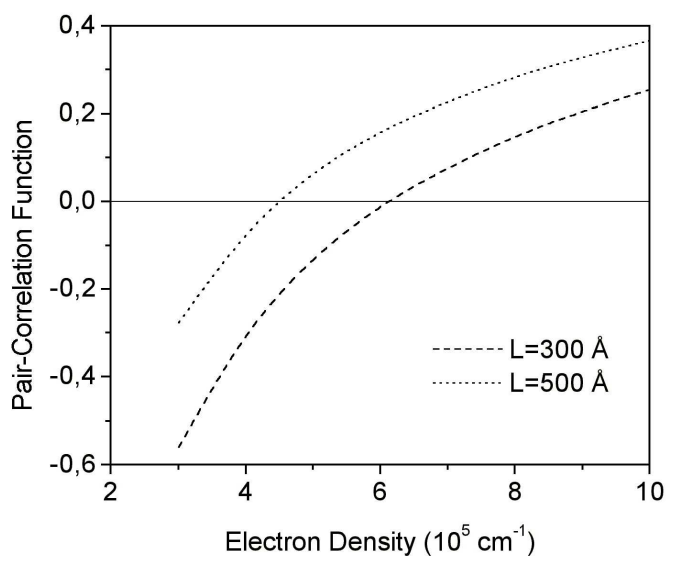

FIG. 2: The pair-correlation function $g(x \approx 0)$, as a function of the electronic density for two wire widths, $\mathrm{L}=300$ ? and $\mathrm{L}=500$ ?.

In Fig. 3 we show the effects of the plasmon- phonon LO interaction on the pair correlation function as a function of the wire width. As we can note increasing the spatial confinement (decreasing the wire width) the polaronic effect became more 
expressive, and can not be neglected in the calculations.

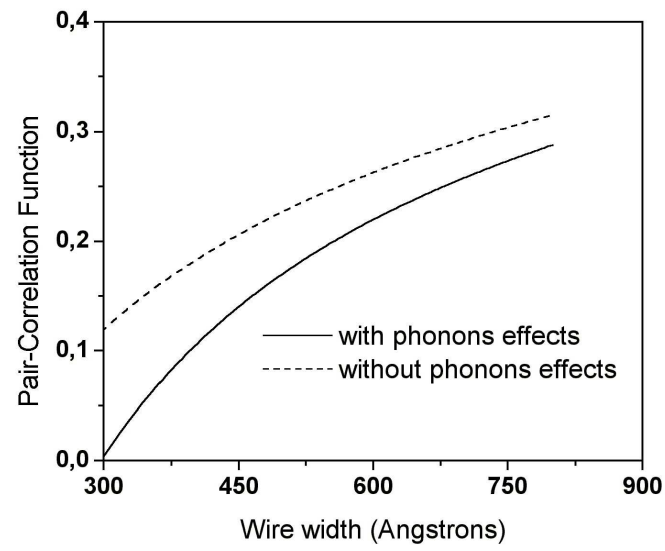

FIG. 3: The pair-correlation function as a function of the wire width with and without the polaronic effects for electronic density $\rho=6.1 \times 10^{5} \mathrm{~cm}^{-1}$

\section{CONCLUSION}

We have calculated the intrasubband pair-correlation function for a quasi-one-dimensional electron gas confined in a
GaAs-AlGaAs rectangular quantum wire with infinite potential barrier height within the Random-Phase Approximation. In this calculation the effects of the electron - LO phonon interaction on the critical densities in quasi-one-dimensional systems were considered. We have studied the dependence of the pair-correlation function with the electronic density, with and without the polaronic contribution. We have found the electronic densities values where $\mathrm{g}(\mathrm{x})$ assumes positive values for small interparticle separation. We also have verified that the critical density increases with decreasing wire width and as more realistic the model of calculation as more inadequate is the RPA approach to calculate the collective excitation energies

\section{Acknowledgement}

We thank to the Fundação de Apoio à Pesquisa FUNAPE UFG and Pró-Reitoria de Pesquisa e Pós-Graduação da UCG (PROPE) for financial support.

[1] K. Putteneers, F. Brosens, S. N. Klimin, and J. T.Devreese, Solid State Comm. 135, 108 (2005).

[2] S. N. Klimin and J. T. Devreese, Physical Rev. B68, 245303 (2003).

[3] V. B. Campos, Marcos H. Degani, and O. Hipólito Solid State Comm. 79, 473 (1991).

[4] A. N. Borges, F. A. P. Osório, and P. C. M. Machado, Microelectronics Journal 34, 529 (2003).

[5] L. Wendler, R. Haupt, and R. Pechstedt, Phys. Rev. B43, 14669 (1991).

[6] A. N. Borges, F. A. P. Osório, P. C. M. Machado, and O. Hipólito , Modern. Phys. Lett. B 13, 819 (1999).

[7] D. W. Wang, A. J. Millis, and S. Das Sarma, Solid State Comm 131, 637 (2004).

[8] A. R. Goñi, A. Pinczuck, J. S. Weiner, J. M. Calleja, B. S. Den- nis, L. N. Pfeiffer, and K. W. West, Phys. Rev. Lett. 67, 3298 (1991).

[9] A. N. Borges, M. H. Degani, and O. Hipólito, Superlat.and Microstruc. 13, 51 (1993).

[10] A. Gold and A. Gazali, Phys. Rev. B41, 7626 (1990); ibid 41, 8318 (1991).

[11] A. N. Borges, O. Hipólito, and S. A. Leão, Phys. Rev. B15, 4680 (1997).

[12] P. C. M. Machado, J. R. Leite, F. A. P. Osório, and A. N. Borges Phys. Rev. B56, 4128 (1997).

[13] K. S. Singwi, M. P. Tosi, R. H. Land, and A. Sjolander, Phys. Rev. 176, 589 (1968).

[14] J. B. B. da Cunha, J. F. R. da Cunha, P. C. M. Machado, F. A. P. Osório, and A. N. Borges, Braz. Journ. Phys. 34, 699 (2004). 